\title{
Tratamento do aneurisma da aorta toracoabdominal com endoprótese ramificada para as artérias viscerais
}

\author{
Branched endovascular stent graft for thoracoabdominal \\ aortic aneurysm repair
}

\author{
André Simi ${ }^{1}$, Renato Ishii ${ }^{1}$, Marcelo Ferreira ${ }^{2}$, Anelise Santos ${ }^{3}$, Antonio Carlos Simi ${ }^{4}$
}

\section{Resumo}

Apresentamos um caso de aneurisma da aorta toracoabdominal (AATA) tratado, exclusivamente, pela técnica endovascular, utilizando uma endoprótese ramificada e customizada.

Paciente do sexo feminino, 68 anos de idade, tabagista, hipertensa, portadora de extenso AATA e múltiplas comorbidades que restringiam a indicação de cirurgia convencional. $\mathrm{O}$ aneurisma iniciava-se na aorta torácica descendente, estendendo-se até a aorta abdominal infra-renal, envolvendo as emergências das artérias viscerais, tronco celíaco, artérias mesentérica superior e renais. O AATA foi tratado pela técnica endovascular com implante de uma endoprótese ramificada. Essa endoprótese ramificada foi customizada com base nas características anatômicas da aorta e no posicionamento dos ramos viscerais, obtidos em angiotomografia, objetivando excluir o aneurisma, mantendo a perfusão das artérias viscerais. O procedimento foi realizado em centro cirúrgico, sob anestesia combinada, regional e geral, antecedido de drenagem liquórica e sob orientação fluoroscópica. O acesso para o implante do corpo principal da endoprótese ramificada e o controle radiológico foram realizados através das artérias femorais, previamente dissecadas. Através das ramificações da endoprótese, foram implantadas extensões secundárias, com stents revestidos, para as respectivas artérias viscerais, cujo acesso foi realizado via artéria axilar esquerda. O tempo total do procedimento foi de 14 horas, com 4 horas e 30 minutos de fluoroscopia, e foram utilizados $120 \mathrm{~mL}$ de contraste iodado.

No pós-operatório, a paciente apresentou instabilidade hemodinâmica. Ecocardiograma transesofágico mostrou dissecção retrógrada da aorta torácica, tipo A, seguida de trombose espontânea da falsa luz. A tomografia de controle mostrou exclusão do AATA e perviedade das pontes para os ramos viscerais, sem vazamentos. A alta ocorreu no $13^{\circ}$ dia de pós-operatório.

O tratamento endovascular do AATA com endoprótese ramificada é factível. A melhora dos recursos técnicos e da qualidade dos materiais poderá ampliar a indicação desse procedimento como alternativa à cirurgia aberta.

Palavras-chave: Aneurisma toracoabdominal, endoprótese ramificada, artérias viscerais.

\begin{abstract}
We report a case of branched stent graft system for endovascular repair of thoracoabdominal aortic aneurysm (TAAA).

A 68-year-old female patient, smoker, hypertensive, with a large TAAA and multiple comorbid conditions that restricted indication for conventional surgery. The aneurysm originated from the descending thoracic aorta, extending until the infrarenal abdominal aorta, involving the emergence of visceral arteries, celiac trunk, superior and renal mesenteric arteries. The TAAA was treated with the endovascular technique using a branched stent graft. This stent graft was customized based on the anatomical characteristics of the aorta and on the position of visceral branches, which were obtained by tomographic angiography, with the aim of excluding the aneurysm and maintaining perfusion of visceral arteries. The procedure was performed under regional and general anesthesia in the surgical room, preceded by cerebrospinal fluid drainage under fluoroscopic guidance. The femoral arteries, which were previously dissected, were used to implement the branched stent graft and for radiological control. Through the stent graft branches, secondary extensions were implanted, with covered stents, to the respective visceral arteries, which were approached via left axillary artery. Total operative time was 14 hours, 4 hours and 30 minutes of fluoroscopy time and $120 \mathrm{~mL}$ of iodinated contrast.
\end{abstract}

The patient became hemodynamically unstable after the surgery. Transesophageal echocardiogram showed a type A retrograde dissection of the thoracic aorta, followed by spontaneous thrombosis of the false lumen. Control tomography showed exclusion of the TAAA and patency of the bypasses to visceral branches, with no endoleaks. The patient was discharged on the $13^{\text {th }}$ postoperative day.

Branched endovascular stent graft for TAAA repair is feasible. Improvements in techniques and materials may increase the indication of branched stent graft as an alternative to open surgery.

Keywords: Thoracoabdominal aneurysm, branched stent graft, visceral arteries.

1. Cirurgião vascular. Preceptor, Especialização em Cirurgia Vascular e Endovascular, Hospital Santa Helena, São Paulo, SP.

2. Chefe, Serviço Integrado de Técnicas Endovasculares (SITE), Rio de Janeiro, RJ.

3. Médica residente, Cirurgia Vascular e Endovascular, Hospital Santa Helena, São Paulo, SP.

4. Chefe, Serviço de Cirurgia Vascular e Endovascular, Hospital Santa Helena, São Paulo, SP.

Artigo submetido em 24.10.06, aceito em 28.02.07.

J Vasc Bras 2007;6(1):86-91.

Copyright $\odot 2007$ by Sociedade Brasileira de Angiologia e de Cirurgia Vascular. 


\section{Introdução}

O aprimoramento dos materiais e das técnicas de tratamento endovascular tornou possível o reparo com sucesso dos aneurismas da aorta toracoabdominal (AATA) utilizando uma endoprótese ramificada, a qual, implantada na aorta, permite a exclusão do aneurisma e a revascularização das artérias viscerais: artéria mesentérica superior, tronco celíaco e artérias renais. Trata-se de uma endoprótese customizada, cujos desenho e construção são específicos para cada paciente. $\mathrm{O}$ objetivo é relatar o nosso primeiro caso de AATA tratado com essa nova tecnologia.

\section{Descrição do caso}

Paciente do sexo feminino, de 68 anos, tabagista, portadora de hipertensão arterial sistêmica, insuficiência cardíaca congestiva, doença pulmonar obstrutiva crônica e insuficiência renal controlada, foi encaminhada para tratamento de AATA. O diagnóstico foi realizado através de ecocardiograma transesofágico e tomografia de tórax e abdômen, realizados após episódios de queda e perda da consciência. No exame físico, apresentava-se hemodinamicamente compensada. Palpava-se massa pulsátil abdominal, com presença de pulsos femorais e distais e ausência de sopros. A angiotomografia mostrava aorta tortuosa e ateromatosa em toda sua extensão, com dilatação aneurismática iniciando-se na aorta descendente e estendendo-se até o segmento infra-renal, envolvendo as artérias viscerais. $\mathrm{O}$ colo proximal do aneurisma media $3 \mathrm{~cm}$ de diâmetro na aorta descendente, e o diâmetro máximo era de 6,1 $\mathrm{cm}$ acima da emergência do tronco celíaco, com extenso trombo mural concêntrico ao longo da dilatação aneurismática, com espessura de até $2,5 \mathrm{~cm}$ (Figura 1). O preparo técnico para a confecção da endoprótese ramificada - suas dimensões e o posicionamento dos ramos para as respectivas pontes - foi determinado e baseado pelo estudo e medição das imagens tomográficas. A endoprótese ramificada foi customizada conforme projeto específico para a paciente, tendo a forma de uma ampulheta. Do seu segmento médio, mais estreito que as extremidades, partem as ramificações para as artérias viscerais (Figura 2). O procedimento cirúrgico para implante do dispositivo foi realizado em centro cirúr- gico, sob orientação fluoroscópica, utilizando um arco cirúrgico GE-OEC-9800. A anestesia foi combinada, regional e geral, precedida de drenagem liquórica, com monitorização contínua e heparinização sistêmica. Os acessos arteriais para a realização do procedimento foram obtidos através de incisão transversa suprainguinal bilateral; as artérias femorais foram dissecadas e bainhas $5 \mathrm{~F}$ foram introduzidas via dissecção da artéria axilar esquerda. Pela artéria femoral esquerda, foi introduzido cateter diagnóstico pig tail 5F, e realizou-se uma aortografia panorâmica (Figura 3). Após, foi realizada cateterização seletiva de uma artéria visceral, referencial, para permitir o perfeito posicionamento da endoprótese. A introdução e o implante da endoprótese ramificada (Cook-Zenith), com calibre de $22 \mathrm{~F}$, foram realizados diretamente através da artéria femoral comum direita, sobre uma guia rígida Amplatz 0,035 e sob orientação fluoroscópica. Na artéria femoral esquerda, foi introduzida bainha $12 \mathrm{~F}$, e implantou-se um cateter fenestrado para realização de shunt provisório para a artéria renal esquerda estenosada e profilático de isquemia renal. A seguir, procedeu-se à abertura da endoprótese ramificada e à respectiva acomodação proximal e distal com balão complacente. Foi realizada a angiografia panorâmica de controle (Figura 4). A artéria axilar esquerda já dissecada foi utilizada como acesso para o cateterismo seletivo das artérias renais, mesentérica superior e tronco celíaco, e realizou-se a sucessiva revascularização dessas artérias através do implante de extensões pelas ramificações. A primeira artéria revascularizada foi a artéria renal direita. Foi implantada pela ramificação correspondente uma extensão com stent revestido Fluency 8 x $40 \mathrm{~mm}$ e reforço interno com um Wall Stent $10 \times 68 \mathrm{~mm}$. Após a retirada do shunt provisório, foi cateterizada a artéria renal esquerda. $\mathrm{Na}$ artéria renal esquerda, em razão de estenose ostial, foi necessária a colocação inicial de um Wall Stent 10 x $68 \mathrm{~mm}$, seguida de implante pela ramificação de uma extensão com stent revestido Fluency 10 x $40 \mathrm{~mm}$ e reforço interno com Wall Stent 10 x $42 \mathrm{~mm}$. Na seqüência, foi cateterizada seletivamente a artéria mesentérica superior, e, através da ramificação correspondente, foi implantada uma extensão com stent revestido 
Fluency 6 x $60 \mathrm{~mm}$ e reforço interno com Zilver Stent 8 x $40 \mathrm{~mm}$. Finalmente, realizaram-se cateterização do tronco celíaco, implante através da ramificação correspondente de uma extensão com stent revestido Fluency 8 x $80 \mathrm{~mm}$ e reforço interno com um Zilver Stent 10 x 60 $\mathrm{mm}$. O controle angiográfico mostrou posicionamento adequado do corpo principal da endoprótese e das ramificações (Figura 5). O procedimento foi finalizado com arteriorrafia das artérias femorais e axilar com fios de polipropileno 6-0 e sutura das incisões de pele com fios de nylon 5-0.

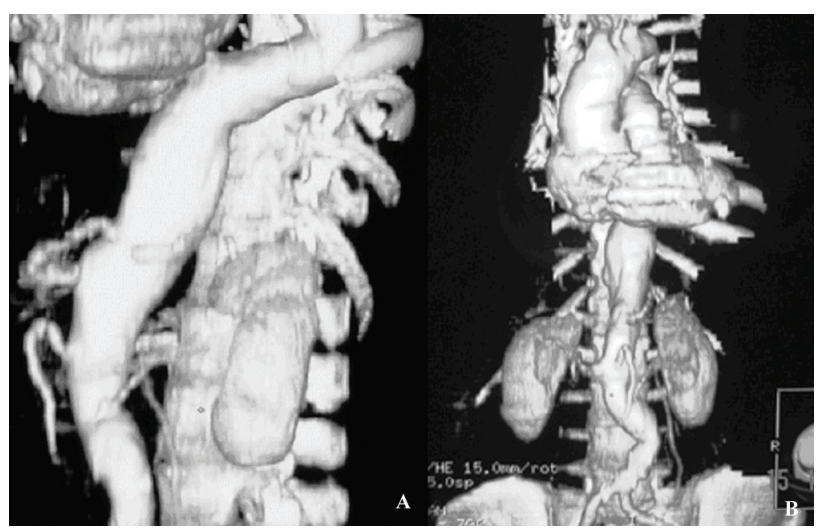

Figura 1 - A angiotomografia pré-operatória em incidência de perfil (A) e em ântero-posterior (B) mostra extenso aneurisma toracoabdominal envolvendo as artérias viscerais
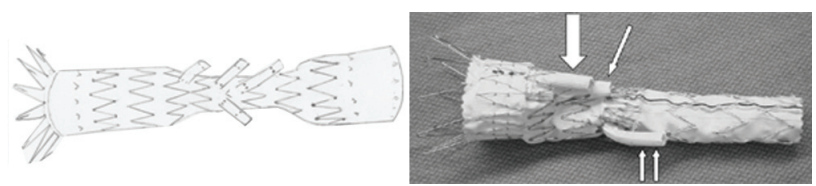

Figura 2 - Esquema ilustrativo da planta da endoprótese ramificada e exemplo fotográfico de uma endoprótese ramificada

A duração total do procedimento foi de 14 horas. Foram necessárias 4 horas e 30 minutos de fluoroscopia. A paciente recebeu $120 \mathrm{~mL}$ de substância de contraste e duas bolsas de sangue.

O pós-operatório (PO) foi realizado em unidade de tratamento intensivo. No segundo dia, houve piora dos padrões hemodinâmicos. $\mathrm{O}$ raio $\mathrm{X}$ de tórax mostrou alargamento do mediastino. A paciente foi mantida com infusão em bomba de noradrenalina, entubação

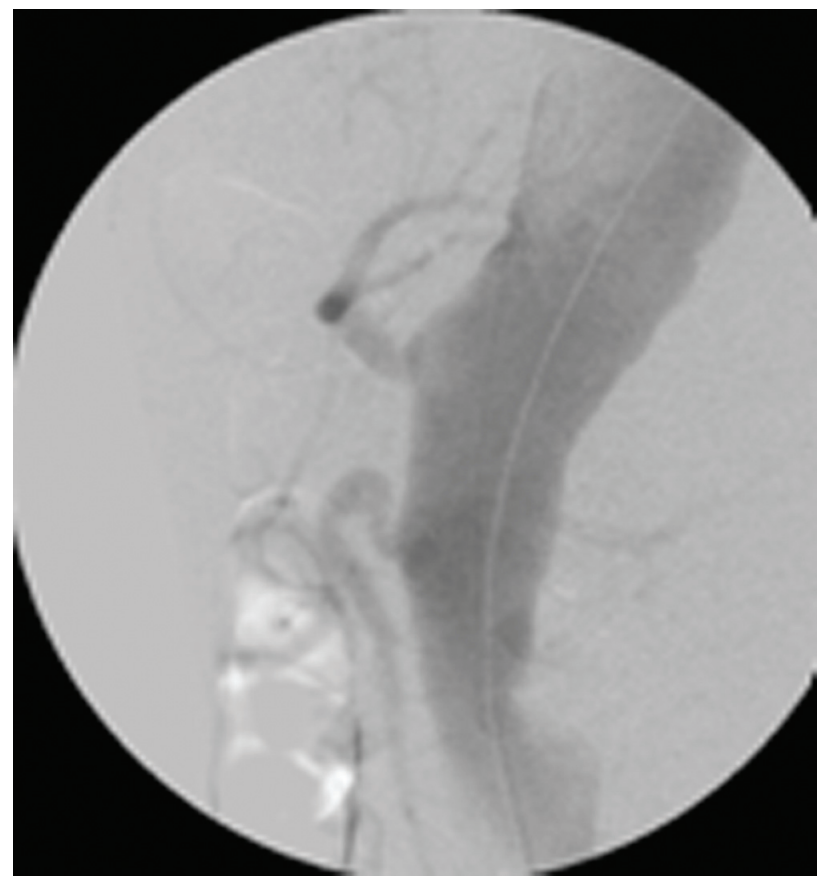

Figura 3 - Aortografia intra-operatória em perfil, mostrando aneurisma toracoabdominal envolvendo os ramos viscerais

orotraqueal e sedada. Novo ecocardiograma transesofágico revelou que um segmento da aorta torácica estava dissecado, proximal à emergência da artéria subclávia esquerda, estendendo-se até $3 \mathrm{~cm}$ do plano valvar. O pericárdio estava normal. A hemoglobina não se alterou, e a creatinina era de 1,5.

Optou-se pela manutenção do tratamento clínico, com controle tensional e nutrição enteral. A paciente apresentou balanço hidroeletrolítico positivo e função renal normal. No terceiro dia, o ecocardiograma transesofágico de controle mostrou trombose total da falsa luz. A paciente foi mantida com noradrenalina e dobutamina. No quarto dia, foi suspensa a dobutamina, com redução da sedação, e a paciente foi extubada. No quinto dia, apresentou episódio de febre, leucocitose e icterícia. TGO e TGP normais, aumento de FAA, de gamaglutamil-transpeptidade (GGT) e creatinina de 1,4. Introduziu-se cefepime $4 \mathrm{~g} / \mathrm{dia}$, metronidazol 1,5 $\mathrm{g} /$ dia e vancomicina $2 \mathrm{~g} /$ dia. Realizou-se ultra-som de abdômen de controle sem alterações patológicas. A paciente foi mantida em unidade de tratamento intensivo até o $10^{\circ}$ dia de PO. Na enfermaria, apresentou 


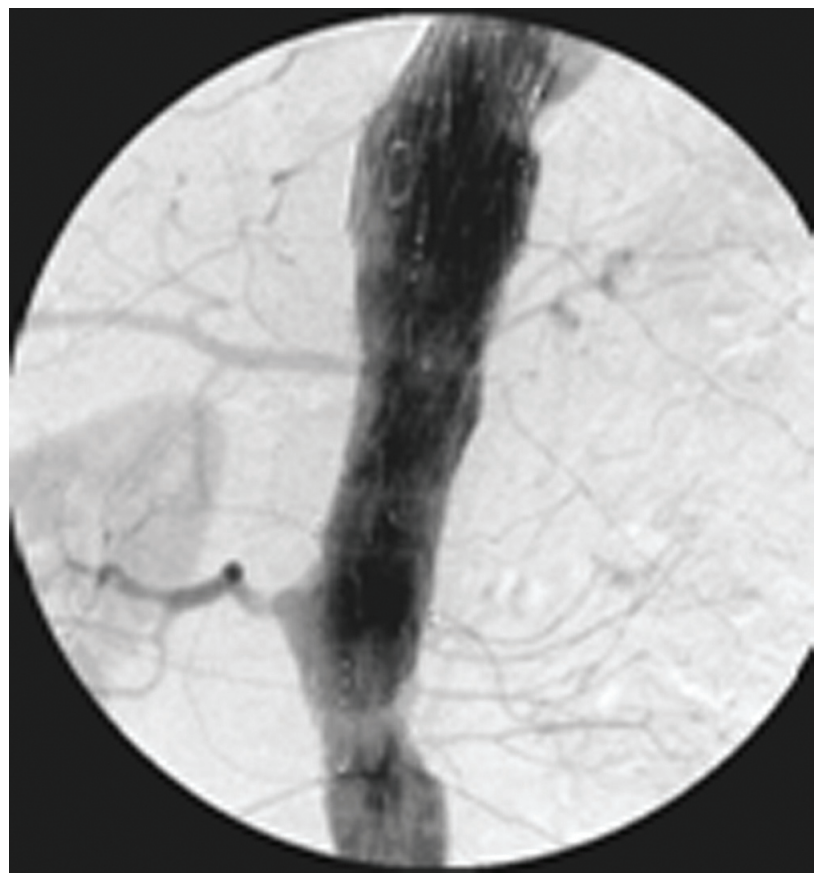

Figura 4 - Angiografia após liberação do corpo principal da endoprótese, mostrando bom posicionamento do dispositivo, ausência de vazamentos e manutenção da perfusão visceral

melhora progressiva da icterícia, boa função renal e alimentação via oral. No $12^{\circ}$ dia de $\mathrm{PO}$, foi suspenso o metronidazol. No $13^{\circ}$ dia, foi submetida a angiotomografia de controle, que mostrou perfeita perviedade da endoprótese principal e dos ramos secundários, sem sinais de vazamento (Figura 6). Recebeu alta em boas condições.

\section{Discussão}

A idéia do tratamento endovascular das lesões arteriais não é nova. Dotter, em 1969, realizou as primeiras experiências de tratamento endovascular com sucesso em artérias poplíteas de cães ${ }^{1}$. Mas somente em 1991 Parodi relatou os primeiros casos de tratamento endovascular de aneurisma da aorta infra-renal em humanos com sucesso ${ }^{2}$. A indicação dessa modalidade de tratamento tem limitações e está relacionada às condições anatômicas dos aneurismas ${ }^{3,4}$, mas vem se ampliando, progressivamente, em razão do aprimoramento e desenvolvimento das técnicas, dos materiais e equipamentos utilizados na realização desses procedimentos. As dificuldades para o emprego dessa técnica

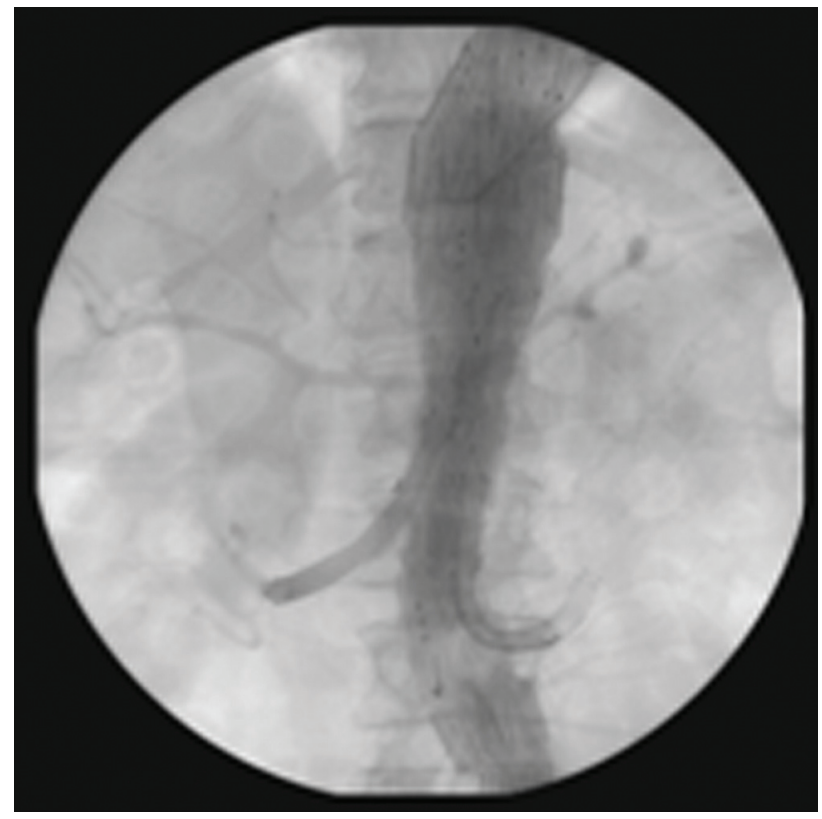

Figura 5 - Angiografia intra-operatória de controle após implantação da endoprótese principal e das respectivas pontes, sem vazamentos e com perfeita perviedade dos ramos viscerais

são maiores quando o aneurisma é extenso, envolvendo a aorta torácica e abdominal, com comprometimento das artérias viscerais e renais, e continuam sendo um grande desafio para o cirurgiões ${ }^{5,6}$. Convencionalmente, os AATA são tratados através de toracotomias, com amplas incisões no abdômen e no tórax ${ }^{7}$. Objetivando minimizar esses procedimentos, tem-se preconizado o tratamento combinado, também denominado de híbrido. Essa técnica utiliza uma endoprótese tubular, permitindo a exclusão do aneurisma da aorta torácica e do segmento que envolve a origem das artérias viscerais, e acesso abdominal aberto, permitindo a realização de pontes extra-anatômicas para as respectivas artérias viscerais ${ }^{8}$. Recente publicação mostrou bons resultados iniciais com essa técnica9 ${ }^{9}$ Entretanto, o mais recente avanço no tratamento do AATA é o seu reparo realizado com técnica exclusivamente endovascular. Para a realização desse procedimento, é necessária uma endoprótese especial, ramificada, específica e customizada de acordo com as características anatômicas da aorta do paciente. A endoprótese ramificada tem um corpo principal e ramificações secundárias correspondentes às artérias viscerais que serão revascularizadas. A grande 
vantagem da técnica é permitir o acesso e a realização do procedimento através de pequenas incisões periféricas. Além disso, todo o procedimento é realizado sem a interrupção total do fluxo arterial na aorta e nos ramos viscerais ${ }^{10}$. Existem marcas radiopacas nas extremidades superior e inferior da endoprótese, ao longo da sua superfície anterior e nas ramificações, que servem como guia de orientação proximal, distal e axial. O corpo principal da endoprótese ramificada é mais estreito que as suas extremidades e não alcança totalmente a parede da aorta, dilatada e com trombos, permitindo o manuseio das respectivas ramificações. Trata-se de um conceito novo, mas constitui, ainda, uma experiência limi$\operatorname{tada}^{11,12}$.

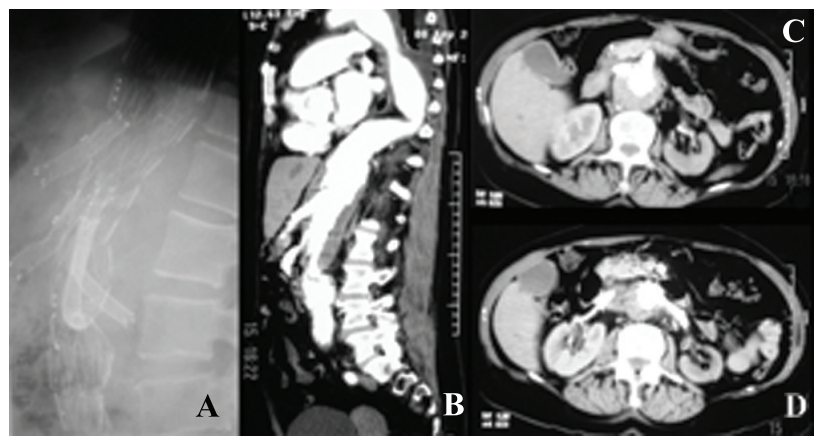

Figura 6 - Controle radiológico pós-operatório: A) raio $\mathrm{X}$ simples mostrando a prótese locada; B) angiotomografia com reconstrução, corte coronal mostrando perviedade da endoprótese principal e seus ramos; C e D) angiotomografia em cortes transversais mostrando perviedade do corpo principal da endoprótese e dos ramos viscerais (mesentérica superior, tronco celíaco e renais)

A nossa experiência no tratamento endovascular dos aneurismas da aorta foi seqüencial: tratamento endovascular dos aneurismas da aorta abdominal infrarenal; tratamento dos aneurismas da aorta torácica e dissecções; tratamento concomitante do aneurisma da aorta torácica com endoprótese tubular e do aneurisma abdominal infra-renal com endoprótese bifurcada, sem cobrir o segmento da aorta correspondente à emergência das artérias viscerais. Finalmente, realizamos o tratamento endovascular desse caso, cobrindo todo o segmento do AATA com o implante do corpo principal da endoprótese ramificada e as respectivas pontes secundárias para as artérias viscerais. A possibilidade de excluir o aneurisma com segurança, sem clampeamento e sem comprometer totalmente a perfusão distal e visceral, constitui, sem dúvida, um procedimento atrativo e menos invasivo. Toda a instrumentação, introdução e liberação do corpo principal da endoprótese e o implante das pontes secundárias para os ramos da aorta foram realizados sob orientação fluoroscópica. Somente o acesso arterial foi cirúrgico, feito através pequenas incisões nas regiões inguinal e axilar, distantes da doença-alvo a ser tratada. Eventualmente, alguns fatores podem prejudicar a perfeita realização dessa técnica: a excessiva tortuosidade das artérias ilíacas, que limitam a liberdade do sistema de rotação e navegação dos cateteres; a presença de trombos espessos e ostiais; as estenoses ostiais das artérias viscerais; e também, dependendo do tamanho do aneurisma, o espaço existente entre a endoprótese e a emergência da artéria visceral pode dificultar a inserção das pontes secundárias e suas extensões. No nosso caso, houve necessidade de tunelização de um trombo parietal espesso, para permitir o acesso ao óstio de uma artéria visceral. Foi necessária, ainda, a colocação de um stent ostial na artéria renal esquerda estenosada, previamente ao implante da ramificação.

A indicação cada vez mais freqüente da aplicação de técnicas endovasculares não se restringe mais ao entusiasmo dos cirurgiões ${ }^{13}$. As previsões de Collin ${ }^{14}$, de que o reparo endovascular de aneurismas era um experimento falho, não se confirmaram. A evidente melhora dos resultados é fruto das novas tecnologias. Os avanços progressivos no tratamento endovascular dos aneurismas em todos os segmentos da aorta e a crescente melhora nos resultados justificam a indicação dessa técnica em pacientes selecionados e de risco mais elevado. O tratamento do AATA com endoprótese ramificada não constitui, ainda, um tratamento de rotina. Como demonstramos em nosso caso, o tratamento é individual e específico para cada paciente. Depende de uma endoprótese especialmente projetada e customizada para cada paciente, de acordo com os dados anatômicos obtidos em tomografia e angiotomografia de ótima resolução. O sucesso desse procedi- 
mento permite concluir que a técnica é factível e deve ser considerada como mais uma alternativa no tratamento dos AATA.

\section{Agradecimento}

Ao Dr. Timothy Chuter, pela colaboração no projeto e implante da endoprótese.

\section{Referências}

1. Dotter C. Tranluminally-placed coilspring endarterial tube grafts. Long-term patency in canine popliteal artery. Invest Radiol. 1969;4:329-32.

2. Parodi JC, Palmaz JC, Barone HD. Transfemoral intraluminal graft implantation for abdominal aortic aneurysms. Ann Vasc Surg. 1991;5:491-9.

3. Makaroun MS, Dillavou ED, Kee ST, et al. Endovascular treatment of thoracic aneurysms: results of the phase II multicenter trial of the GORE TAG thoracic endoprosthesis. J Vasc Surg. 2005;41:1-9.

4. Neuhauser B, Czermak BV, Fish J, et al. Type A dissection following endovascular thoracic aortic stent-graft repair. J Endovasc Ther. 2005;12:74-81.

5. Dake MD, Miller DC, Semba CP, Mitchell RS, Walker PJ, Liddell RP. Transluminal placement of endovascular stent-grafts for the treatment of descending thoracic aortic aneurysms. N Engl J Med. 1994;331:1729-34.

6. Brandt M, Hussel K, Walluscheck KP, Boning A, Rahimi A, Cremer J. Early and long-term results of replacement of the descending aorta. Eur J Vasc Endovasc Surg. 2005;30:365-9.
7. Svensson LG, Crawford ES, Hess KR, Coselli JS, Safi HJ. Experience with 1509 patients undergoing thoracoabdominal operations. J Vasc Surg. 1993;17:357-68; discussion 368-70.

8. Coselli JS, Conklin LD, LeMaire SA. Thoracoabdominal aortic aneurysm repair: review and update of current strategies. Ann Thorac Surg. 2002;74:S1881-4.

9. Black SA, Wolfe JH, Clark M, Hamady M, Cheshire NJ, Jenkins MP. Complex thoracoabdominal aortic aneurysms: endovascular exclusion with visceral revascularization. J Vasc Surg. 2006;43:1081-9.

10. Chuter TA, Gordon RL, Reilly LM, Goodman JD, Messina LM. An endovascular system for thoracoabdominal aortic aneurysm repair. J Endovasc Ther. 2001;8:25-33.

11. Chuter TA, Howell BA. Suprarenal stents and other advances in endovascular aneurysm repair. Surg Clin North Am. 2004;84:1319-35.

12. Browne TF, Hartley D, Purchas S, Rosenberg M, Van Schie G, Lawrence-Brown M. A fenestrat covered suprarenal aortic stent. Eur J Vasc Endovasc Surg. 1999;18:445-9.

13. Sternbergh WC, Nordness PJ, York JW, Conners MS, Carter G, Money SR. Endo-exuberance to endo-reality: trends in the management of 431 AAA repairs between 1996 and 2002. J Endovasc Ther 2003; 10: 418-23.

14. Collin J, Murie JA. Endovascular treatment of abdominal aortic aneurysm: a failed experiment. $\mathrm{Br} \mathrm{J}$ Surg. 2001;88:1281-2.

Correspondência:

Antonio Carlos Simi

Rua Barata Ribeiro, 414

CEP 01308-000 - São Paulo, SP

E-mail: simimed@terra.com.br 